\title{
Estrategia de planificación turística y las funciones participativas de los stakeholders en la provincia de Los Ríos
}

Tourist planning strategy and the participatory functions of stakeholders in the province of Los Ríos

Christian Rivera García. ${ }^{1}$, Glenda Parra Cerezo. ${ }^{2}$ \& Alicia Zavala Santana. ${ }^{3}$

Recibido: 12-08-2021 / Revisado: 22 -08-2021 /Aceptado: 13-09-2021/ Publicado: 05-11-2021

\begin{abstract}
DOI: https://doi.org/10.33262/concienciadigital.v4i4.1.1981

Introduction. In recent years, the tourism planning strategy of Stakeholders at the international level has begun to rotate from the administration, it has had a considerable shift in governance, one of the main characteristics, the agents involved in the sector are integrated into the processes, decision-making, management and evaluation of the results of the public administration, expanding its favorable participation and effectiveness in the sector. In Ecuador, the strategic planning of tourism and the functions of the Stakeholders are unified and organized in a line of commercial coexistence, with the use of participatory diagnostic technological tools, becoming a necessity for the optimal development of tourist destinations, these agents are considered important for the creation of new policies and procedures in the tourism field. The objective of this work is to identify tourism planning strategies to define the roles of Stakeholders in these activities. Methodology. The research followed a descriptive methodology, with the use of theoretical methods and documentary review, the deductive method was also used to identify the direct beneficiaries of this research, surveys were applied to 10 professionals
\end{abstract}

\footnotetext{
1 Universidad Técnica de Babahoyo FCJSE, Ecuador. crivera@utb.edu.ec

2 Universidad Técnica de Babahoyo FCJSE, Ecuador. gparra@utb.edu.ec

3 Universidad Técnica de Babahoyo FCJSE, Ecuador. aliciazavalasantana@gmail.com
} 
in the tourism field of the Province of Los Ríos, who stated that they did not know the actors that have to do with the tourist activity identified in a strategic tourism plan. Results. Tourism in the Province of Los Ríos is a sector that has been positioning itself as a destination irregularly, since the tourist attractions, tourist plant do not provide the conditions to provide a service according to tourist requirements. Conclusions. The limited notion of administration in the public sector towards the locality has produced little emphasis on the development of this activity, limiting the population towards other horizons of local and national productivity.

Keywords: tourism planning, governance, favorable participation, functions.

\section{Resumen}

Introducción. En los últimos años la estrategia de planificación turística de los Stakeholders a nivel internacional ha comenzado a girar desde la administración, ha tenido un desplazamiento considerable en la gobernabilidad, una de las principales características, los agentes involucrados en el sector son integrados en los procesos, toma de decisiones, gestiones y evaluación de los resultados de la administración pública, ampliando su participación favorable y efectividad en el sector. En Ecuador la planificación estratégica del turismo y las funciones de los Stakeholders se unifican y organizan en una línea de coexistencia mercantil, con la utilización de herramientas tecnológicas participativas de diagnóstico, convirtiéndose en una necesidad para el desarrollo óptimo de los destinos turísticos, estos agentes son considerados importantes para la creación de nuevas políticas, y gestiones en el ámbito turístico. El Objetivo de este trabajo consiste en identificar las estrategias de planificación turística para definir las funciones de los Stakeholders en estas actividades. Metodología. La investigación siguió una metodología descriptiva, con la utilización de métodos teóricos y la revisión documental, también se utilizó el método deductivo para identificar a los beneficiarios directos de esta investigación, se aplicaron encuestas a 10 profesionales del ámbito turístico de la Provincia de Los Ríos, que manifestaron desconocer a los actores que tienen que ver con la actividad turística identificada en un plan estratégico de turismo. Resultados. El turismo en la Provincia de Los Ríos es un sector que ha venido posicionándose como un destino de forma irregular, debido a que los atractivos turísticos, planta turística no prestan las condiciones para brindar un servicio acorde a exigencias del turista. Conclusiones. La limitada noción de administración en el sector público hacia la localidad ha producido escaso énfasis en el desarrollo de esta actividad, limitando a la población hacia otros horizontes de productividad local y nacional.

Palabras Clave: planificación turística, gobernabilidad, participación favorable, funciones.

\section{Introducción}

En los últimos años las estrategias de planificación turística de los Stakeholders a nivel internacional ha comenzado a girar desde la administración, esto ha tenido un desplazamiento considerable desde el gobierno hacia la gobernabilidad, una de las 
principales características es que los agentes involucrados en el sector puedan verse inmiscuidos en los procesos, toma de decisiones, gestiones y evaluación de los resultados de la administración pública, consiguiendo una participación favorable y efectividad en el sector.

En América Latina las participaciones de los stakeholders en las estrategias de planificación turística constituyen un proceso primordial que ratifica la gobernanza en el destino turístico, produciendo que los agentes inmersos en este proceso tengan relevancia y presencia en agendas gubernamentales e institucionales a nivel latinoamericano.

En Ecuador la planificación estratégica del turismo y la participación de los Stakeholders con la utilización de herramientas participativas de diagnóstico se ha convertido en una necesidad para el desarrollo óptimo de los destinos turísticos, estos agentes son considerados importantes para la creación de nuevas políticas, y gestiones en el ámbito turístico. En la Provincia de Los Ríos se está implementando en ciertos sectores la funcionalidad participativa de los Stakeholders en los destinos turísticos para el fortalecimiento de la Planificación estratégica del turismo. Estos agentes posibilitan nuevas estrategias para consolidar proyectos que estructuralmente son óptimos, pero con la integración de funciones de los Stakeholders, se prevé una conspiración del igual en el sector turísticos.

La presente investigación trata de identificar y definir las funciones de los Stakeholders en las estrategias de la planificación turística, haciendo una aproximación y análisis descriptivo, el estudio trata de examinar el acercamiento que estos agentes tienen entre sí, sus intereses y las actividades empresariales en las que ellos actúan, para concretar procesos que impulsen un valor molecular en redes sociales de profesionales, clientes sector, público y privado, que además de generar información dentro del mercado, en algunos casos estarán inmersos en los mismos a la hora de desarrollar estrategias y tomar decisiones empresariales.

Las estrategias de planificación turística mediante los Stakeholders como menciona Morales y Hernández (2019) “es importante analizar el entorno de las empresas turísticas para llevar a cabo un proceso de toma de decisiones y gestión estratégica apropiadas", en este sentido para deliberar se necesita analizar a todo el entorno turístico, por ejemplo; las asociaciones empresariales, clientes, accionistas, profesionales entre otros, para poder aliarse y obtener resultados óptimos en integración de objetivos en común. En este proceso, el sector turístico, en mancomunidad, recibe de manera directa e indirectamente beneficios no solo como participantes, sino también prestadores de servicios, y agentes de cambio a la sociedad, organizaciones que trascienden hacia mejoras en la calidad de vida y del entorno en el que están involucrados.

Los stakeholders o grupos de interés, son todos aquellos que afectan la actividad de la organización, pone de manifiesto un contacto con los interesados en la empresa, siendo un activo estratégico y ventaja competitiva para cualquier organización. 
Según Jaramillo et al (2019) la planificación del producto turístico es el resultado del diagnóstico del estado de desarrollo del sistema turístico de un determinado espacio geográfico, por lo que el desarrollo de los mismos es un factor vital para poder poner en valor los atractivos turísticos y que los turistas puedan satisfacer sus expectativas y necesidades, así como abordar de manera sistemática los aspectos de competitividad turística, que conduzcan al debido planeamiento y consolidación del desarrollo de un destino turístico (pág. 34).

La elaboración de un producto turístico da como consecuencia la evolución de un sistema, de un espacio para que los atractivos turísticos sean observados de manera que los visitantes se encuentren satisfechos y alcancen todas sus expectativas, implementar nuevas fortalezas para vencer a la competencia tomando en cuenta las debilidades del mercado meta. González (2017) enfatizan que la planificación es comúnmente entendida como un proceso en que se definen metas y los medios necesarios para alcanzarlas, la planificación debería proveer la información necesaria para la toma de decisiones apropiada, democrática e informada. La palabra planificar se define como un procedimiento en donde se fijan objetivos y por todo el proceso que se debe seguir para que estos sean alcanzados. La planificación consiste en el manejo correcto de la información al momento de la toma de decisión, considera estructuras organizaciones con intereses en común, pero con sistemas de alcance competitivos como los Stakeholders. Desde la óptica Freeman \& Liedtka (1997), menciona que:

Los Stakeholders es una palabra del inglés que, en el ámbito empresarial, significa 'interesado' o 'parte interesada', y que se refiere a todas aquellas personas u organizaciones afectadas por las actividades y las decisiones de una empresa. En toda organización, además de sus propietarios, participan diversos actores claves y grupos sociales que están constituidos por las personas o entes que, de una manera y otra, tienen interés en el desempeño de una empresa porque están relacionadas, bien directa, bien indirectamente, con ella. (pág. 26)

Stakeholder hace referencia a todo individuo o entidad perjudicada por las actividades y las opiniones vertidas dentro de una organización. Sin embargo, intervienen diferentes personajes claves y colectivos sociales que son designados por rangos de jerarquía empresarial con el objetivo de manejar correctamente la empresa, esos enfoques también consideran jerarquías como: los stakeholders primarios que son aquellos imprescindibles para el funcionamiento de la organización, es decir, todos aquellos que tienen una relación económica directa con la empresa, como los accionistas, los clientes o los trabajadores (García-Sánchez \& Noguera-Gámez, 2017).

Los stakeholders primario son fundamentales para el desempeño de la institución, en concreto establecen lasos laborales que beneficien a la empresa económicamente como los socios, propietarios o clientes.

Los stakeholders secundarios, que son aquellos que no participan directamente en las actividades de la empresa, pero que, sin embargo, se ven afectados por ella, como, por ejemplo, los competidores o la comunidad, entre otros, los stakeholders secundarios no 
intervienen personalmente en las actividades de la empresa sin embargo se ven afectados por la misma. Los autores Morales y Hernández (2019) en su trabajo titulado "Los Stakeholders del Turismo" mencionan a los diferentes actores o agentes (stakeholders) que intervienen en la actividad turística, mencionando que el estudio contiene un acercamiento a la definición de stakeholders del turismo, señalando las prioridades que éstos determinan y teniendo en cuenta que cuanto mejor identificados estén, estos stakeholders y sus intereses, más eficaz será la actividad empresarial en la que actúan. Esto nos ayudará a concretar y visualizar los actores implicados en la actividad turística para profundizar, con ello, en el conocimiento sobre la creación, desarrollo e implantación de Sistemas de Inteligencia de Marketing en un territorio turístico (pág. 56).

La actividad turística consiste en un método significativo de stakeholder destacando su énfasis, los mismos que se encuentran establecidos con el objetivo de detectarlos, depende mucho de la actividad mercantil en la que interactúan, ésta sirve de ayuda para precisar y observar los personajes que intervienen en el turismo en la información sobre el desarrollo e implantación de sistemas de inteligencia de marketing en una zona turística, comprendiendo el SIM como un conjunto de procedimientos de recolección de datos, incrementando su valor político a través de redes sociales, anuncios, espacios publicitarios que a su vez establecen información valida que es utilizada al momento de tomar una decisión.

En el artículo titulado "La participación de los stakeholders en los destinos turísticos españoles: análisis de la situación actual", de los autores Gómez y Duque (2015), muestra los resultados del proyecto realizado por la Escuela de Organización Industrial (EOI) y la Universidad Nebrija sobre el modelo de eficiencia del turismo en España. Dentro de este proyecto, uno de los objetivos más importantes fue el de conocer la relevancia que los diversos stakeholders otorgan a su participación en los asuntos públicos en el ámbito de los destinos españoles. En primer lugar, con el fin de lograr este objetivo se recurrió a fuentes de información secundaria que permitieran analizar la relevancia de la participación de los stakeholders en los asuntos públicos, así como las diversas tipologías de participación existentes y los mecanismos o herramientas para llevarlas a cabo. En segundo lugar, a partir de este marco teórico, se consideró necesario conocer la situación actual de los procesos participativos en los destinos turísticos españoles, identificar los principales problemas que los rodean y determinar las posibles líneas de actuación para mejorarlos.

La participación del turismo en la provincia de los Ríos consiste en descubrir la importancia de los diferentes stakeholders que aportan e intervienen con mecanismos o medios para ponerlos en práctica. Los stakeholders de este tipo inciden directamente en la estandarización de las actividades de la organización, representados por algunas instituciones gubernamentales o asociaciones con actividades similares. Las relaciones se establecen de acuerdo con acciones, propósitos o problemas de carácter común como la promoción turística, la capacitación, la calidad en el servicio o la responsabilidad social. 
El parentesco reglamentario se da a conocer por medio de dos formas: la jurídica y la gubernamental. Las relaciones se definen teniendo en cuenta la responsabilidad social. Hace referencia a las opiniones otorgadas por las certificaciones. Sin embargo, los problemas sociales y del medio ambiente que repercute en el turismo, por ejemplo, la contaminación ambiental afecta al hábitat y a los animales, se convierte en un obstáculo. Por esta razón es necesario interactuar con la prensa, grupos de rescate o tribus urbanas con el fin de buscar un beneficio en ambas partes. Sperandio et al. (2019) tienen como objetivo central la identificación de los principales stakeholders de la ruta turística, y como objetivo secundario la evaluación de los medios de junción entre los principales stakeholders identificados, con base en el estudio de la práctica relacional de estos en la posible suposición de incremento de los negocios y mejorías en el desempeño global de la ruta.

Los stakeholders del turismo en Puerto Cayo es un tema de gran relevancia para el crecimiento del pueblo, mediante el presente estudio se identificó los agentes involucrados tanto los primarios como los secundarios, para poder tener una mayor eficacia del con quien se puede trabajar para el desarrollo del turismo en la localidad, así como también una vez identificado se procedió a generar un análisis de los mismos y verificar las falencias y él porque de la poca visita de las personas a la localidad, siendo este un balneario donde el impacto turístico debe ser abundante durante todo el año y no solo en temporadas, haciendo el análisis de esto, se procedió a determinar las potencialidades de un sistema de inteligencia de marketing turístico. Este autor impulsa a imbricar los bienes, servicios, productos y procesos turísticos en la provincia de Los Ríos a través del incremento de la zona turística, llevar a cabo una investigación con el propósito de comprobar la carencia de visitantes y todos sus pormenores, porque por lo general el lugar debe de ser visitado durante todo el año y no solo por estaciones, esto se puede hacer, generando nuevos métodos de marketing para implementar en sectores turísticos, existen diversos factores que participan en las labores turísticas y establecer una proximidad a su delimitación para identificar la capacidad de introducir un sistema inteligente de marketing turístico generando resultados favorables al sector económico (Esteban, 2018).

\section{Metodología}

La investigación cualitativa facilitó la observación del objeto en escenarios fenomenológicos secuenciales en virtud de su aplicación científica. Y el enfoque cuantitativo permitió realizar una investigación de carácter descriptivo. La aplicación de un cuestionario con preguntas cerradas viabilizó información congruente con información dirigida ampliar las posibilidades de cooperación interinstitucional en el sector productivo. El método científico deductivo identificó y focalizó, beneficiarios directos, mientras el método inductivo problematizó la raíz de la gestión en su funcionalidad, detectando un presupuesto valorativo de especulaciones en gobernanza y gobernabilidad institucional-social, con estrategias de sujeción turística en pleno apogeo de recomendaciones sustantivas programáticas. La información documental acercó criterios 
de otros autores al desafío de incluirlos en el análisis de la planificación turística de la provincia.

La planificación impulsa seriamente a los stakeholders al compromiso de transformar y mejorar los bienes, servicios, productos y procesos turísticos., previo la orientación particular para definir el cumplimiento del objetivo impulsado en esta investigación, la participación de los profesionales fue fundamental.

\section{Resultados}

Según los resultados, los stakeholders priorizan la evolución del proceso turístico considerando a la población in situ, el acompañamiento de los emisarios de la función pública, instituciones que vigilan el seguimiento de actividades complementarias con enfoques de control y cumplimiento de normativas que recubren las actividades de gestión para afianzar la relación estrecha de acciones con objetivos mancomunados de gestión territorial en la provincia de Los Ríos.

En este estudio están involucrados elementos claves de la planificación turística que dirigen instancias en el desarrollo de la provincia, es encomiable traducir el objeto de estudio a empresarios, profesionales inmersos en la actividad de recreación como base de una economía circular.

El turismo en la provincia de Los Ríos, paulatinamente se presenta como un impulso en la generación de plazas de trabajo y de oportunidades micros empresariales de progreso, la situación se complica en el momento de manejar esta productividad en períodos claves de generación de recursos económicos, aunque la infraestructura es todavía incierta, la población insiste en supervivir a las condiciones de empleabilidad regional sin la claridad de asesoramiento interno y externo institucional.

La provincia brinda al turista, actividades variadas en especial para el carnaval, adecua las playas en los ríos, fortalece actividades culturales, incita a la práctica de deportes extremos en los cauces de los ríos, entre competencias y recreación en plenitud óptima y seguridad. Es interesante la organización de conciertos al aire libre, ferias gastronómicas, exposición de productos agrícolas de la zona con proyección de emprendimientos comunitarios del producto estrella de la región, el cacao, aunque no sea permeable permanentemente, la actividad de planificación está presente en los niveles de gestión pública y privada: ministerios de turismo, cámara de turismo, entre otros.

Los stakeholders necesitan evidenciar su funcionamiento integral e inclusivo en toda la provincia, realizando puntos expositorios de la potencialidad que tiene la zona sur, centro y norte de la provincia a nivel nacional con la ayuda de los prestadores de servicios: hoteleros, restaurantes, entre otros.

Los agentes recreativos turísticos están sujetos a los cambios temporales climatológicos, políticos, rentas, impuestos, que es deducible de la misma actividad, esta lógica de asimilación en servicios turísticos es el motivo para innovar procesos atractivos de marketing en el sector, planificación que necesita de nuevos escenarios seguros, cómodos 
y confiables de recreación y disfrute, impulsando el crecimiento económico, en diversidad de medios de producción y patrones de consumo integrados al agenda productiva turística del ministerio de turismo y la cámara provincial.

Los servicios proporcionados en la región no responden a las exigencias de los visitantes, por comodidad y hospitalidad en el trato y productos de calidad, síndrome del turismo convencional, extendido a las provincias sin este fenómeno tradicional del turismo, este es el momento donde los stakeholders agudizan funcionamiento extremo de atractividad porcentual de distribución en obligaciones recreativas.

Tabla 1

Los stakeholders y el impulso turístico

\begin{tabular}{lcl}
\hline \multicolumn{1}{c}{ Indicadores dominantes } & $\begin{array}{c}\text { Porcentaje de aceptación } \\
\text { prioritaria }\end{array}$ & $\begin{array}{c}\text { Acciones procedimentales en beneficio } \\
\text { de la creatividad e innovación }\end{array}$ \\
\hline $\begin{array}{l}\text { Los stakeholders que } \\
\text { impulsan la actividad turística } \\
\text { Los stakeholders son }\end{array}$ & $1 \%$ & $\begin{array}{l}\text { Asesoramiento turístico en proyectos } \\
\text { turísticos diversificando la participación }\end{array}$ \\
indispensables para el turismo & $8 \%$ & $\begin{array}{l}\text { Consolidar los bienes, servicios, } \\
\text { productos y procesos turísticos en los } \\
\text { diferentes niveles de la planificación } \\
\end{array}$ \\
& & estratégica. \\
\hline
\end{tabular}

La operación turística esta esquematizada en el conjunto de bienes, servicios productos y procesos turísticos embrionados en la participación comunitaria, y de las bases amplias que pregonan sistemas de planificación de multiniveles entre la empresa pública y en mejoramiento de las estructuras del marketing turístico, despegando procesos de fortalecimiento del turismo tradicional e impulsando nuevos tipos de turismo en complementariedad de la hospitalidad poblacional desde un enfoque de asociatividad y convivencia turística.

Se conceptualizó el alcance y significado de la responsabilidad social, sobre los grupos de atención prioritaria, que exista compromiso y obligación de los individuos involucrados para formar un sistema integral de Estrategias turísticas que fomenten el turismo en la provincia de Los Ríos.

Tarea ineludible, buscar aliados estratégicos, que afirmen el compromiso de elaborar estrategias de planificación turística para mejorar las funciones participativas de los Stakeholders en la provincia de Los Ríos, mejorando las relaciones empresariales, fortaleciendo a la provincia como destino turístico reconocido a nivel nacional.

Es importante que exista una integración de funciones con los Stakeholders, que puedan motivar la satisfacción de todos, con fiel compromiso de satisfacer necesidades comunitarias, inspirando la participación en la toma de decisiones sobre nuevas actividades empresariales. 


\section{Conclusiones}

- Las estadísticas de este estudio confieren criterios de complementariedad empresarial, pública y privada, el cuestionario diseñado cumplió con el objetivo de acercar a la realidad turística a los encuestados y facilitar eslabones productivos en reacción poblacional y generacional

- El impacto que propician los nuevos profesionales dentro de la economía circular como asesores bilaterales de la empresa pública y privada, constituye elemento crucial desde los stakeholders en base a conocimientos triangulares actitudinales, procedimentales y cognitivos, que impulsan la integración y participación directa en la planificación estratégica del turismo regional, buscando la diversidad en la empresa conjunta como símbolo de progreso comunitario local.

- La diversidad de servicios que comprende el producto turístico (alojamiento, restauración, transporte, información turística clave) hace que éstos sean suministrados mediante un sistema de planificación turística para mejorar las funciones de los Stakeholders en sinergia a favor de la provincia de Los Ríos involucrando a las autoridades, academia y otros actores sociales en pleno acompañamiento de talleres y capacitaciones congruentes con la idiosincrasia del sector.

- Es necesario implementar un sistema de planificación turística para el crecimiento comercial y económico del sector, importante que los Stakeholders se involucren dentro de las charlas sobre las normativas de inversión, estos agentes pueden incrementar las inversiones mediante la venta de un imagen en perfeccionamiento económico, político y financiero con credibilidad y confianza productiva nacional e internacional, en equilibrio de condiciones óptimas para el inversionista y la población local, regional y nacional

\section{Referencias bibliográficas}

Esteban Santos, L., García-Medina, I., \& Bellido-Pérez, E. (2018). Comunicación y moda en el entorno digital: comportamiento del público seguidor de blogueras de moda./ANABEL/Downloads/3805-Texto\%20del\%20art\%C3\%ADculo-8656-110-20191226.pdf

Freeman, E. \& Liedtka, J. (1997). Capitalismo de stakeholders y cadena de valor. Revista europea de gestión, 15 (3), 286-296.García. (2017). Los Stakeholders Primarios y Secundarios.

García - Sánchez, IM \& Noguera - Gámez, L. (2017). Informes integrados y participación de las partes interesadas: el efecto sobre la asimetría de la información. Responsabilidad Social Corporativa y Gestión Ambiental, 24 (5), 395-413. 
Gómez, \& Duque. (2015). La participación de los stakeholders en los destinos turísticos españoles: análisis de la situación actual. Aposta Revista de Ciencias Sociales, 151-174. https://www.redalyc.org/pdf/4959/495950261007.pdf

González Moyano, D. A. (2017). Apoyo a la implementación de las actividades establecidas en los programas formulados en el sistema de gestión ambiental del Ministerio de Comercio, Industria y Turismo.

Jaramillo, J. N., Rosero, D. C., Rosales, H. B. \& Pérez, L. C. (2019). Las actividades agroturísticas y el desarrollo turístico en la finca San Vicente. SATHIRI, 14(1), 186-194. Salma. (2019). Los Stakeholders del Turismo.

Morales, \& Hernández. (2019). Los Stakeholders del Turismo. Book of proceedings vol. I - international conference on tourism \& management studies - algarve 2011(ISSN: 2182-8458), 894-903.

Sperandio, Zapparoli, Munhoz, \& Dorion. (2019). Los stakeholders de influencia directa en el desarrollo de la ruta turística de una localidad en la sierra gaucha: un estudio exploratorio. XV international conference on industrial.

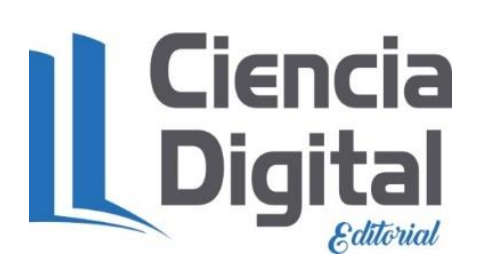




\section{PARA CITAR EL ARTÍCULO INDEXADO.}

Rivera García, C., Parra Cerezo, G., \& Zavala Santana, A. (2021). Estrategia de planificación turística y las funciones participativas de los stakeholders en la provincia de $\begin{array}{llll}\text { Los Ríos. } & \text { ConcienciaDigital, } & 4(4.1), & 178-188 .\end{array}$ https://doi.org/10.33262/concienciadigital.v4i4.1.1981

\section{\Ciencia}

El artículo que se publica es de exclusiva responsabilidad de los autores y no necesariamente reflejan el pensamiento de la Revista Conciencia Digital.

El artículo queda en propiedad de la revista y, por tanto, su publicación parcial y/o total en otro medio tiene que ser autorizado por el director de la Revista Conciencia Digital.

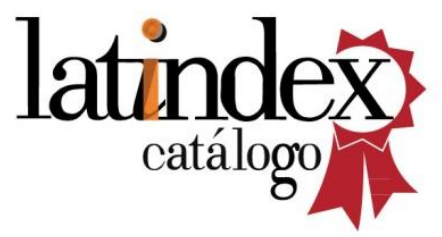

